\title{
ENERGY-MOMENTUM SCHEME FOR NONLINEAR THERMO-ELECTRO-ELASTODYNAMICS
}

\author{
Marlon Franke $^{1 *}$, Rogelio Ortigosa ${ }^{2}$, Antonio J. Gil ${ }^{3}$ and Moritz Hille ${ }^{1}$ \\ ${ }^{1}$ Karlsruhe Institute of Technology - Institute of Mechanics, Otto-Ammann-Platz 9, 76131 Karlsruhe, \\ Germany, \{marlon.franke,peter.betsch\}@kit.edu, http://www.ifm.kit.edu \\ 2 Technical University of Cartagena - Computational Mechanics, Scientific Computing Group, 30202 \\ Cartagena, Spain, rogelio.ortigosa@upct.es, www.upct.es/mc3/en/dr-rogelio-ortigosa \\ ${ }^{3}$ Swansea University, Zienkiewicz Centre for Computational Engineering, Fabian Way, Swansea, SA1 \\ 8EN, United Kingdom, a.j.gil@ swansea.ac.uk and www.swansea.ac.uk/staff/engineering/a.j.gil
}

Key words: Coupled problems, thermo-electro-elastodynamics, finite deformations, energy-momentum scheme, finite element method

\begin{abstract}
The present contribution aims at the consistent discretisation of nonlinear, coupled thermoelectro-elastodynamics. In that regard, a new one-step implicit and thermodynamically consistent energymomentum integration scheme for the simulation of thermo-electro-elastic processes undergoing large deformations will be presented. The consideration is based upon polyconvexity inspired, constitutive models and a new tensor cross product algebra, which facilitate the design of the so-called discrete derivatives (for more information it is referred to the pioneering works [3, 2]). The discrete derivatives are fundamental for the algorithmic evaluation of stresses and other derived variables like entropy density or the absolute temperature leading to a structure preserving integration scheme. In particular, recently published works of the authors concerning consistent time integration of large deformation thermo-elastodynamics (see [6]) and electro-elastodynamics (see [11]) are combined to a unified integration scheme. Numerical computations demonstrate the stability and conservation properties of the proposed energy-momentum scheme.
\end{abstract}

\section{INTRODUCTION}

The continuous increase of computer power within the last decades enables more realistic simulations, e.g. coupled cardiac or soft robotic simulations employing fine discretization in space and time, without the necessity of making major simplifications. In such simulations, large displacements/rotations and deformations, as well as nonlinear thermodynamic and electrostatic effects can be essential to properly reflect the reality. In addition, coupled problems are developed for a wide range of applications. For instance, the high actuation possibilities of dielectric elastomers (DEs) or electroactive polymers (EAPs) require thermo-electro-mechanical coupled simulations. However, a consistent time discretization of these systems poses new challenges.

EAPs and DEs are typically modeled as coupled systems (cf. [13]) and are particularly sensitive to changes of the thermal field, but remain frequently unconsidered (cf. $[19,18,16,5])$. The continuum 
mechanical formulation of EAPs under anisothermal conditions has been investigated (cf. [17]) In this connection, also numerical studies have recently been published (see e.g. [10] and the references therein), which, however, are limited to both the mechanical and thermal static cases, i.e. any time effects are not considered.

Energy-momentum (EM) time integration schemes consistently reproduce the conservation properties of continua (cf. [9]) in the time discrete setting. In particular, when using EM schemes the discrete counterparts of the balance of linear, angular momentum and total energy of the system are in agreement with the laws of thermodynamics for the system at hand. In this context, [1] gives an overview of the development of structure-preserving time integrators in the field of computational mechanics. The recent work [2] is considered to be pioneering a new family of structure-preserving integrators in the framework of polyconvex elasticity (cf. [15]). Therein, three discrete gradients replace the traditional projection-based discrete gradient. This formulation was extended to the field of thermoelasticity in $[6,12]$ and to the field of electroelasticity in $[11,7]$. Compared to classical projection-based discrete gradients (cf. [9]), the new algorithmic stress formulas in [2, 6, 11, 7, 12] adopt a particularly simple structure. Moreover, the mixed 4- and 10-field formulations in [11, 7] further simplify the construction of energy- and angular momentum consistent integrators. A crucial factor for this simplification is the use of both the tensor cross product and the polyconvexity inspired constitutive modeling.

To the best knowledge of the authors, for all works concerning electro-thermo-mechanics dealing with finite deformations, thermal and electrical fields energy-consistent numerical treatment has not been elaborated yet. The aim of this work is to bridge this gap, i.e. to construct an EM time integration scheme

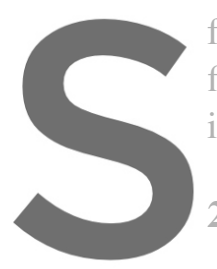
for the field of finite ele

first three authors concern itself shows to be very rob

2 Kinematies
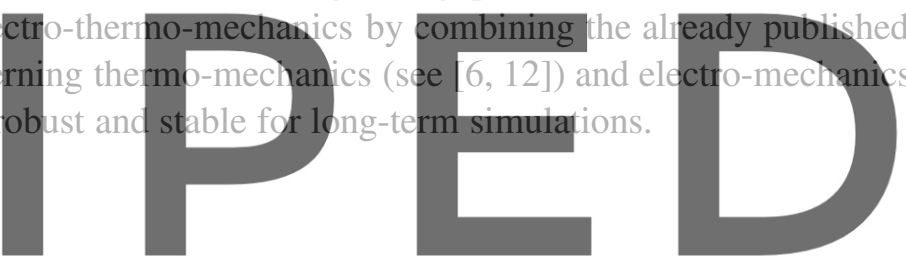

A deformable, thermo-electro-mechanical continuum body with reference configuration $\mathcal{B}_{0}$ and current

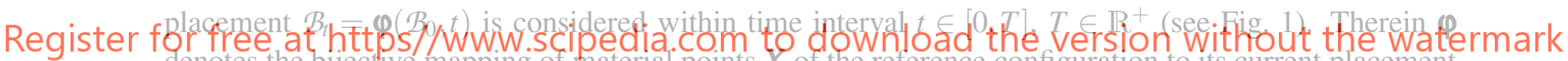
denotes the bijective mapping of inaterial points $X$ of the reference configuration to its current placement

via $x=\varphi:=\varphi(X, t)$ (see Fig. 1). The boundary $\partial \mathcal{B}$ of the body is subdivided into non overlapping mechanical Dirichlet and Neumann boundaries $\partial_{\varphi} \mathcal{B}_{0}, \partial_{T} \mathcal{B}_{0}$, thermal Dirichlet and Neumann boundaries $\partial_{\phi} \mathcal{B}_{0}, \partial_{\omega} \mathcal{B}_{0}$ and electrical Dirichlet and Neumann boundaries $\partial_{Q} \mathcal{B}_{0}, \partial_{\theta} \mathcal{B}_{0}$, respectively, i.e.

$$
\begin{aligned}
\partial_{\boldsymbol{\varphi}} \mathcal{B}_{0} \cup \partial_{\boldsymbol{T}} \mathcal{B}_{0}=\partial \mathcal{B}_{0} & =\partial_{\phi} \mathcal{B}_{0} \cup \partial_{\omega} \mathcal{B}_{0} & \partial_{\boldsymbol{\varphi}} \mathcal{B}_{0} \cap \partial_{\boldsymbol{T}} \mathcal{B}_{0}=\emptyset & =\partial_{\phi} \mathcal{B}_{0} \cap \partial_{\omega} \mathcal{B}_{0} \\
& =\partial_{Q} \mathcal{B}_{0} \cup \partial_{\theta} \mathcal{B}_{0} & & =\partial_{Q} \mathcal{B}_{0} \cap \partial_{\theta} \mathcal{B}_{0}
\end{aligned}
$$

According to Fig. 1, the following mappings for fibres $\mathrm{d} \boldsymbol{x} \in \mathcal{B}_{t}, \mathrm{~d} \boldsymbol{X} \in \mathcal{B}_{0}$, area elements $\mathrm{d} \boldsymbol{a} \in \mathcal{B}_{t}, \mathrm{~d} \boldsymbol{A} \in \mathcal{B}_{0}$ and volume elements $\mathrm{d} v \in \mathcal{B}_{t}, \mathrm{~d} V \in \mathcal{B}_{0}$ are introduced as

$$
\mathrm{d} \boldsymbol{x}=\boldsymbol{F} \mathrm{d} \boldsymbol{X}, \quad \mathrm{d} \boldsymbol{a}=\boldsymbol{H} \mathrm{d} \boldsymbol{A}, \quad \mathrm{d} v=J \mathrm{~d} V
$$

Therein, the deformation gradient $\boldsymbol{F}$, its co-factor $\boldsymbol{H}$ and its determinant $J$ are given by

$$
\boldsymbol{F}=\partial_{\mathbf{X}} \boldsymbol{\varphi}(\boldsymbol{X}, t), \quad \boldsymbol{H}=\operatorname{cof}(\boldsymbol{F})=\frac{1}{2} \boldsymbol{F} \rtimes \boldsymbol{F}, \quad J=\operatorname{det}(\boldsymbol{F})=\frac{1}{6}(\boldsymbol{F} \rtimes \boldsymbol{F}): \boldsymbol{F}
$$




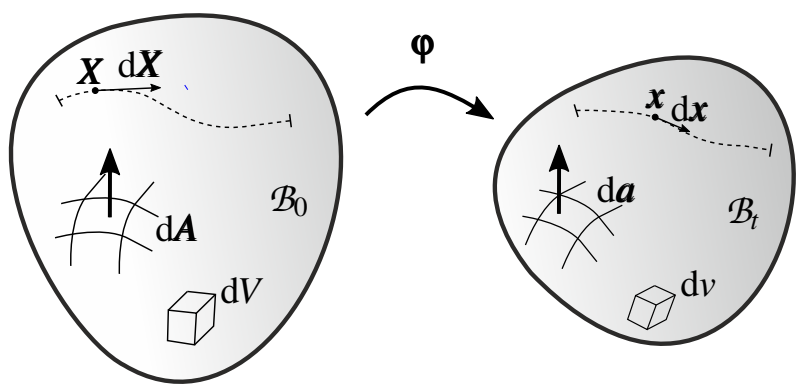

Figure 1: Fibres, area and volume elements for reference and current configuration

For the last two equations, the so-called tensor cross product (for more information it is referred to $[4,3])$ has been used. Assume to have two tensors of second order $\boldsymbol{A}, \boldsymbol{B} \in \mathbb{R}^{3 \times 3}$, the tensor cross product is defined as

$$
(\boldsymbol{A} \rtimes \boldsymbol{B})_{i j}=\varepsilon_{i \alpha \beta} \varepsilon_{j a b} A_{\alpha a} B_{\beta b}
$$

\section{where $\varepsilon_{i j k}$ denotes the Levi-Civita symbol and summation convention applies to pairs of repeated indices.}

By further introducing the second order tensors $\boldsymbol{C}, \boldsymbol{D} \in \mathbb{R}^{3 \times 3}$ the following properties can be applied

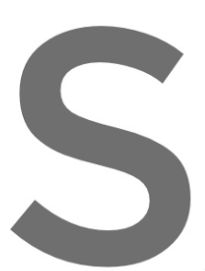

$$
A \times B=B \times A
$$

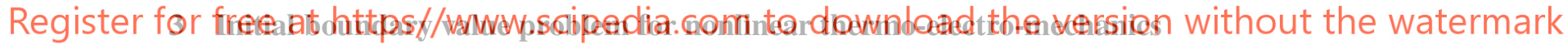

The initial boundary value problem (IBVP) for the considered nonlinear, thermo-electro-mechanic system is comprised of an elastodynamic, an electrostatic and a thermodynamic part. The IBVP for the elastodynamic part is given by the local balance of linear momentum

$$
\rho_{0} \ddot{\boldsymbol{\varphi}}=\operatorname{Div}(\boldsymbol{F} \boldsymbol{S})+\boldsymbol{B}
$$

in $\mathcal{B}_{0}$, where $\rho_{0}$ denotes the reference mass density, $\boldsymbol{S}$ the second PK stress tensor and $\boldsymbol{B}$ the volume load. The corresponding boundary conditions are

$$
(\boldsymbol{F} \boldsymbol{S}) \boldsymbol{N}=\overline{\boldsymbol{T}} \text { on } \partial_{\boldsymbol{T}} \mathcal{B}_{0}, \quad \boldsymbol{\varphi}=\overline{\boldsymbol{\varphi}} \text { on } \partial_{\boldsymbol{\varphi}} \mathcal{B}_{0}
$$

where $\boldsymbol{N}$ denotes the unit outward normal on $\partial_{\boldsymbol{T}} \mathcal{B}_{0}, \overline{\boldsymbol{T}}$ the prescribed surface stress on the mechanical Neumann boundary and $\bar{\varphi}$ the prescribed configuration onto the mechanical Dirichlet boundary. Furthermore, the initial conditions

$$
\boldsymbol{\varphi}(t=0)=\boldsymbol{\varphi}_{0}, \quad \dot{\boldsymbol{\varphi}}(t=0)=\boldsymbol{v}_{0}
$$


in $\mathcal{B}_{0}$ need to be prescribed with initial values for configuration $\boldsymbol{\varphi}_{0}$ and velocity $\boldsymbol{v}_{0}$. Considering Maxwell's equations and neglecting both, magnetic and temporal effects, the special case of electrostatics remains. The boundary value problem of electrostatics is given by Gauß law

$$
\operatorname{Div} \boldsymbol{D}-\rho_{0}^{e}=0 \text { in } \mathcal{B}_{0}, \quad \boldsymbol{D} \cdot \boldsymbol{N}=-\bar{\omega}_{0}^{e} \text { on } \partial_{\omega} \mathcal{B}_{0}
$$

where the Lagrangian electrical displacement vector $\boldsymbol{D}$ and the electric volume charge density $\rho_{0}^{e}$ are introduced. Also, $\bar{\omega}_{0}^{e}$ is the prescribed electric surface charge per unit of an undeformed area of the electrical Neumann boundary. Moreover, Faraday's law is given by

$$
\boldsymbol{E}=-\partial_{\mathbf{X}} \phi \text { in } \mathcal{B}_{0}, \quad \phi=\bar{\phi} \text { on } \partial_{\phi} \mathcal{B}_{0}
$$

where $\boldsymbol{E}$ denotes the Lagrangian electrical field and $\phi$ the electrical potential. Also, $\bar{\phi}$ is the prescribed electrical field onto the electrical Dirichlet boundary. Furthermore, the system is supplemented by the IBVP for thermodynamics given by the local energy balance

$$
\theta \dot{\eta}+\operatorname{Div} Q-R=\frac{d}{d t}(\theta \eta)-\dot{\theta} \eta+\operatorname{Div} Q-R=0
$$

in $\mathcal{B}_{0}$, where the absolute temperature $\theta:=\theta(\boldsymbol{X}, t)$, the entropy density $\eta$, the Piola heat flux vector $\boldsymbol{Q}$ and the heat source density $R$ are introduced. Corresponding to the local energy balance, the following boundary conditions

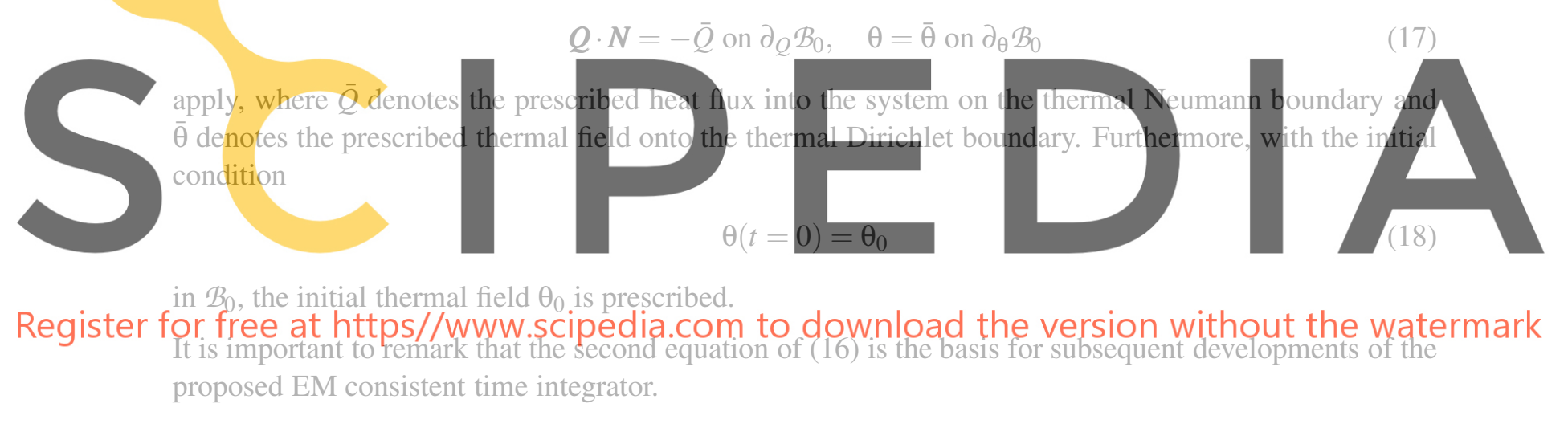

\section{Constitutive modelling}

For the system under consideration, the following Helmholtz free energy is assumed

$$
\Psi=\Psi(\boldsymbol{F}, \boldsymbol{E}, \theta)
$$

Due to the principle of material frame-indifference, it is possible to state

$$
\Psi(\boldsymbol{F}, \boldsymbol{E}, \theta)=\widetilde{\Psi}(\boldsymbol{C}, \boldsymbol{E}, \theta)
$$

with the right Cauchy-Green strain tensor $\boldsymbol{C}=\boldsymbol{F}^{\mathrm{T}} \boldsymbol{F}$. However, we prefer to work with an alternative energy functional denoted as $\widetilde{W}(\boldsymbol{C}, \boldsymbol{D}, \theta)$ which depends upon the electric displacement field $\boldsymbol{D}$, related with the previous Helmholtz functional through the following Legendre transformation, i.e.

$$
\widetilde{\Psi}(\boldsymbol{C}, \boldsymbol{E}, \theta)=\widetilde{W}(\boldsymbol{C}, \boldsymbol{D}, \theta)-\boldsymbol{E} \cdot \boldsymbol{D}
$$


For convenience, it is possible to re-write $\widetilde{W}(\boldsymbol{C}, \boldsymbol{D}, \theta)$ in terms of an extended set of arguments $\mathcal{V}=$ $\{\boldsymbol{C}, \boldsymbol{G}, C\}$ as

$$
W(\mathcal{V}, \boldsymbol{D}, \theta)=\widetilde{W}(\boldsymbol{C}, \boldsymbol{D}, \theta)
$$

where the elements of the extended set are

$$
\boldsymbol{C}=\boldsymbol{F}^{\mathrm{T}} \boldsymbol{F}, \quad \boldsymbol{G}=\operatorname{cof}(\boldsymbol{C})=\frac{1}{2} \boldsymbol{C} \rtimes \boldsymbol{C}, \quad \boldsymbol{C}=\operatorname{det}(\boldsymbol{C})=\frac{1}{3} \boldsymbol{G}: \boldsymbol{C}
$$

It is important to remark that $W$ is not convex with respect to all its dependencies but facilitates the design of an EM scheme. With the tensor product at hand, the time derivative of $\boldsymbol{C}$, its co-factor $\boldsymbol{G}$ and its determinant $C$ are given by

$$
\begin{aligned}
\dot{\boldsymbol{C}} & =\frac{\mathrm{d}}{\mathrm{d} t}\left(\left(\partial_{\mathbf{X}} \boldsymbol{\varphi}\right)^{\mathrm{T}} \partial_{\mathbf{X}} \boldsymbol{\varphi}\right)=\left(\partial_{\mathbf{X}} \dot{\boldsymbol{\varphi}}\right)^{\mathrm{T}} \partial_{\mathbf{X}} \boldsymbol{\varphi}+\left(\partial_{\mathbf{X}} \boldsymbol{\varphi}\right)^{\mathrm{T}} \partial_{\mathbf{X}} \dot{\boldsymbol{\varphi}} \\
\dot{\boldsymbol{G}} & =\frac{\mathrm{d}}{\mathrm{d} t} \frac{1}{2} \boldsymbol{C} \nless \boldsymbol{C}=\boldsymbol{C} \dot{\boldsymbol{C}} \\
\dot{C} & =\frac{\mathrm{d}}{\mathrm{d} t} \frac{1}{3} G: C=G: \dot{C}
\end{aligned}
$$

Accordingly, the time derivative of the energy functional gives

$$
\dot{W}(\mathcal{V}, D, \theta)=S: \frac{1}{2} \dot{C}+E \cdot \dot{D}-\eta \dot{\theta}
$$

where the 2nd PK stress tensor, the electric field and the entropy density
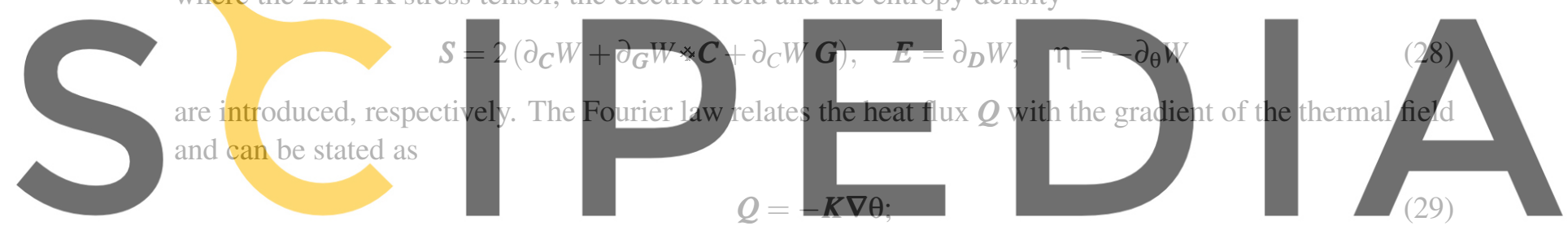

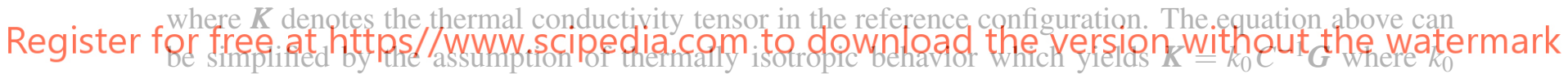

denotes the isotropic coefficient of thermal conductivity.

\section{Weak form}

For the present system, the weak form is given by

$$
\begin{aligned}
& \mathcal{W}_{\boldsymbol{v}}=\int_{\mathcal{B}_{0}}(\boldsymbol{v}-\dot{\boldsymbol{\varphi}}) \cdot \rho_{0} \boldsymbol{w}_{\boldsymbol{v}} \mathrm{d} V=0 \\
& \mathcal{W}_{\boldsymbol{\varphi}}=\int_{\mathcal{B}_{0}} \rho_{0} \dot{\boldsymbol{v}} \cdot \boldsymbol{w}_{\boldsymbol{\varphi}} \mathrm{d} V+\int_{\mathcal{B}_{0}} \boldsymbol{S}: \frac{1}{2} D \boldsymbol{C}\left[\boldsymbol{w}_{\boldsymbol{\varphi}}\right] \mathrm{d} V-\int_{\mathcal{B}_{0}} \boldsymbol{B} \cdot \boldsymbol{w}_{\boldsymbol{\varphi}} \mathrm{d} V-\int_{\partial_{\boldsymbol{T}} \mathcal{B}_{0}} \boldsymbol{T} \cdot \boldsymbol{w}_{\boldsymbol{\varphi}} \mathrm{d} A=0 \\
& \mathcal{W}_{\boldsymbol{\phi}}=\int_{\mathcal{B}_{0}} \boldsymbol{D} \cdot \boldsymbol{\nabla} w_{\phi} \mathrm{d} V+\int_{\mathcal{B}_{0}} \rho_{0}^{\mathrm{e}} w_{\phi} \mathrm{d} V+\int_{\partial_{\omega} \mathcal{B}_{0}} \omega_{0}^{\mathrm{e}} w_{\phi} \mathrm{d} A=0 \\
& \mathcal{W}_{\boldsymbol{D}}=\int_{\Omega_{0}}(\boldsymbol{E}+\nabla \varphi) \cdot \boldsymbol{w}_{\boldsymbol{D}} \mathrm{d} V=0 \\
& \mathcal{W}_{\theta}=\int_{\mathcal{B}_{0}}\left(\frac{d}{d t}(\theta \eta)-\dot{\theta} \eta\right) w_{\theta} \mathrm{d} V-\int_{\mathcal{B}_{0}} \boldsymbol{Q} \cdot \boldsymbol{\nabla} w_{\theta} \mathrm{d} V-\int_{\mathcal{B}_{0}} R w_{\theta} \mathrm{d} V-\int_{\partial_{Q} \mathcal{B}_{0}} Q w_{\theta} \mathrm{d} A=0
\end{aligned}
$$


where solution $\{\boldsymbol{v}, \boldsymbol{\varphi}, \phi, D, \theta\} \in\left\{\mathbb{V}^{\boldsymbol{\varphi}}, \mathbb{V}^{\boldsymbol{\varphi}}, \mathbb{V}^{\phi}, \mathbb{V}^{\boldsymbol{D}, \boldsymbol{w}_{\boldsymbol{D}}}, \mathbb{V}^{\theta}\right\}$ and test functions $\left\{\boldsymbol{w}_{\boldsymbol{v}}, \boldsymbol{w}_{\boldsymbol{\varphi}}, w_{\boldsymbol{\varphi}}, \boldsymbol{w}_{\boldsymbol{D}}, w_{\theta}\right\} \in\left\{\mathbb{V}^{\boldsymbol{w}_{\boldsymbol{\varphi}}}\right.$, $\left.\mathbb{V}^{w_{\varphi}}, \mathbb{V}^{w_{\phi}}, \mathbb{V}^{\boldsymbol{w}_{D, w_{D}}}, \mathbb{V}^{w_{\theta}}\right\}$ the spaces

$$
\begin{aligned}
\mathbb{V}^{\boldsymbol{\varphi}} & =\left\{\boldsymbol{\varphi} \in H^{1}\left(\mathcal{B}_{0}\right) \mid \boldsymbol{\varphi}=\overline{\boldsymbol{\varphi}} \text { on } \partial_{\boldsymbol{\varphi}} \mathcal{B}_{0} \text { and } \boldsymbol{J}>0\right\}, & & \mathbb{V}^{\boldsymbol{w}_{\boldsymbol{\varphi}}}=\left\{\boldsymbol{w}_{\boldsymbol{\varphi}} \in H^{1}\left(\mathcal{B}_{0}\right) \mid \boldsymbol{w}_{\boldsymbol{\varphi}}=\mathbf{0} \text { on } \partial_{\boldsymbol{\varphi}} \mathcal{B}_{0}\right\} \\
\mathbb{V}^{\phi} & =\left\{\phi \in H^{1}\left(\mathcal{B}_{0}\right) \mid \phi=\bar{\phi} \text { on } \partial_{\phi} \mathcal{B}_{0}\right\}, & & \mathbb{V}^{\phi}=\left\{w_{\phi} \in H^{1}\left(\mathcal{B}_{0}\right) \mid w_{\phi}=0 \text { on } \partial_{\phi} \mathcal{B}_{0}\right\} \\
\mathbb{V}^{\theta} & =\left\{\boldsymbol{\theta} \in H^{1}\left(\mathcal{B}_{0}\right) \mid \theta=\overline{\boldsymbol{\theta}} \text { on } \partial_{\theta} \mathcal{B}_{0}\right\}, & & \mathbb{V}^{\theta}=\left\{\theta \in H^{1}\left(\mathcal{B}_{0}\right) \mid w_{\theta}=0 \text { on } \partial_{\theta} \mathcal{B}_{0}\right\} \\
\mathbb{V}^{\boldsymbol{D}, \boldsymbol{w}_{\boldsymbol{D}}} & =\left\{\boldsymbol{D}, \boldsymbol{w}_{\boldsymbol{D}} \in \mathbb{L}_{2}\left(\mathcal{B}_{0}\right)\right\} & &
\end{aligned}
$$

are introduced. In the following, the conservation laws are shown. By setting $\boldsymbol{w}_{\boldsymbol{\varphi}}=\boldsymbol{\xi}=$ const. in the above weak form (31) finally yields the balance of linear momentum

$$
\dot{L}-F^{\text {ext }}=\mathbf{0}
$$

where $\boldsymbol{F}^{\text {ext }}$ denotes the total external force comprised of volume and surface loads. Setting $\boldsymbol{w}_{\boldsymbol{\varphi}}=\boldsymbol{\xi} \times \boldsymbol{\varphi}$ with $\xi=$ const. in (31) yields the balance of angular momentum

$$
j-M^{\text {ext }}=0
$$

where $M^{\text {ext }}$ denotes the total external torque comprised of volume and surface torques. Setting $w_{\varphi}=\xi=$ const. in (32) leads to the global form of Gauß law

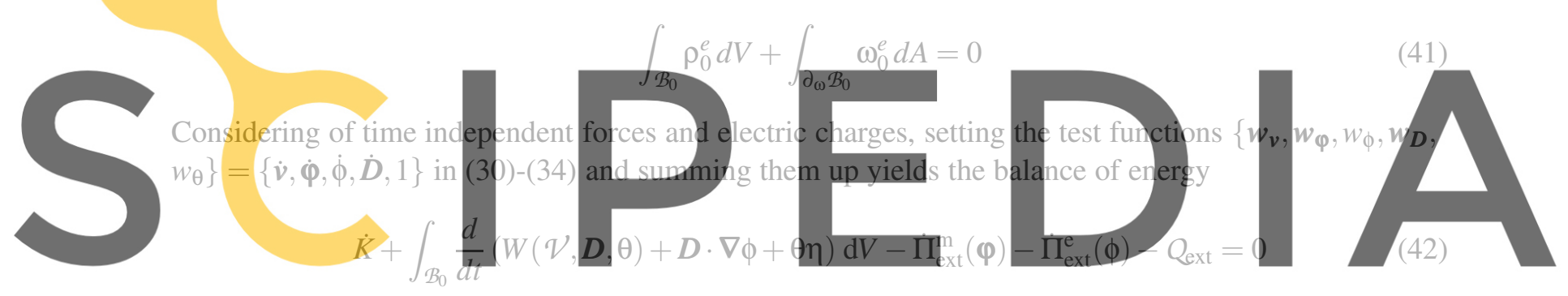

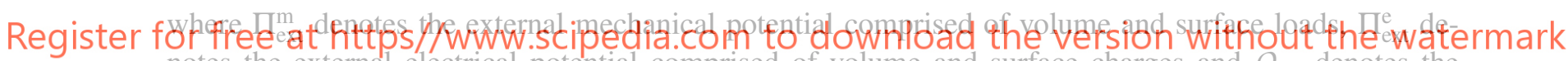 notes the external electrical potential comprised of volume and surface charges and Qext denotes the thermal power.}

\section{Energy-momentum scheme}

In order to apply structure preserving integration the concept of the discrete gradient in the sense of [9] is used to carry out the following steps for time discretisation of (30)-(34)

- substitution of time rates $(\bullet)$ with $\frac{\Delta(\bullet)}{\Delta t}$, where $\Delta(\bullet)=(\bullet)_{n+1}-(\bullet)_{n}$

- midpoint evaluation of terms, namely $(\bullet)_{n+\frac{1}{2}}=\frac{1}{2}\left((\bullet)_{n+1}+(\bullet)_{n}\right)$

- substitution of the derivatives $\left\{\partial_{C} W, \partial_{G} W, \partial_{C} W, \partial_{D} W, \partial_{\theta} W\right\}$ with partitioned discrete gradients $\left\{D_{C} W, D_{G} W, D_{C} W, D_{D} W, D_{\theta} W\right\}$, where the definition of the partioned discrete derivatives are presented in detail in [8]

- fulfillment of the directionality property

$$
D_{\boldsymbol{C}} W: \Delta \boldsymbol{C}+D_{\boldsymbol{D}} W \cdot \Delta \boldsymbol{D}+D_{\theta} W \Delta \theta=\Delta W
$$


Applying these steps to the weak form (30)-(34) yields the semi-discrete system

$$
\begin{aligned}
& \mathcal{W}_{\boldsymbol{v}}^{\text {algo }}=\int_{\mathcal{B}_{0}}\left(\boldsymbol{v}_{n+\frac{1}{2}}-\frac{\Delta \boldsymbol{\phi}}{\Delta t}\right) \cdot \rho_{0} \boldsymbol{w}_{\boldsymbol{v}} \mathrm{d} V \\
& \mathcal{W}_{\boldsymbol{\varphi}}^{\mathrm{algo}}=\int_{\mathcal{B}_{0}} \rho_{0} \frac{\Delta \boldsymbol{v}}{\Delta t} \cdot \boldsymbol{w}_{\boldsymbol{\varphi}} \mathrm{d} V+\int_{\mathcal{B}_{0}} \boldsymbol{S}_{\text {algo }}: \frac{1}{2}\left(\boldsymbol{D}\left[\boldsymbol{w}_{\boldsymbol{\varphi}}\right]\right)_{\mathrm{algo}} \mathrm{d} V-\int_{\mathcal{B}_{0}} \boldsymbol{B}_{n+\frac{1}{2}} \cdot \boldsymbol{w}_{\boldsymbol{\varphi}} \mathrm{d} V-\int_{\partial_{t} \mathcal{B}_{0}} \boldsymbol{T}_{n+\frac{1}{2}} \cdot \boldsymbol{w}_{\boldsymbol{\varphi}} \mathrm{d} A \\
& \mathcal{W}_{\phi}^{\text {algo }}=\int_{\mathcal{B}_{0}} \boldsymbol{D}_{n+\frac{1}{2}} \cdot \boldsymbol{\nabla} \delta \phi \mathrm{d} V+\int_{\mathcal{B}_{0}} \rho_{0}^{e}{ }_{n+\frac{1}{2}} \delta \phi \mathrm{d} V+\int_{\partial_{\omega} \mathcal{B}_{0}} \omega_{0}^{e}{ }_{n+\frac{1}{2}}^{e} \delta \phi \mathrm{d} A \\
& \mathcal{W}_{\boldsymbol{D}}^{\text {algo }}=\int_{\mathcal{B}_{0}} \delta \boldsymbol{D} \cdot\left(\boldsymbol{E}_{\text {algo }}+\nabla \varphi_{n+\frac{1}{2}}\right) \mathrm{d} V \\
& \mathcal{W}_{\theta}^{\text {algo }}=\int_{\mathcal{B}_{0}}\left(\frac{\Delta(\theta \eta)}{\Delta t}-\frac{\Delta \theta}{\Delta t} \eta_{\text {algo }}\right) w_{\theta} \mathrm{d} V-\int_{\mathcal{B}_{0}} \boldsymbol{Q}_{n+\frac{1}{2}} \cdot \nabla w_{\theta} \mathrm{d} V-\int_{\mathcal{B}_{0}} R_{n+\frac{1}{2}}{ }^{w_{\theta}} \mathrm{d} V-\int_{\partial_{Q} \mathcal{B}_{0}} Q_{n+\frac{1}{2}}{ }^{w_{\theta}} \mathrm{d} A
\end{aligned}
$$

where the careful choices

$$
\begin{aligned}
& \boldsymbol{S}_{\text {algo }}=2\left(D_{C} W+D_{G} W * \boldsymbol{C}_{\text {algo }}+D_{C} W \boldsymbol{G}_{\text {algo }}\right), \quad \boldsymbol{E}_{\text {algo }}=D_{D} W, \quad \eta_{\text {algo }}=-D_{\theta} W \\
& \boldsymbol{C}_{\text {algo }}=\boldsymbol{C}_{n+\frac{1}{2}}, \quad \boldsymbol{G}_{\text {algo }}=\frac{1}{3}\left(\boldsymbol{C}\left(\boldsymbol{\varphi}_{n+\frac{1}{2}}\right) * \boldsymbol{C}\left(\boldsymbol{\varphi}_{n+\frac{1}{2}}\right)+\boldsymbol{G}\left(\boldsymbol{\varphi}_{n+\frac{1}{2}}\right)\right)
\end{aligned}
$$

enable to fulfil the directionality property. To verify the conservation properties, the discrete forms of the balance laws are considered. Setting $w_{\varphi}=\xi=$ const. in the above weak form (45) yields the semi-

discrete balance of linear

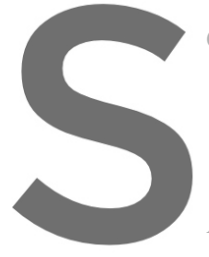

Accordingly, for vanishing external forces $F_{n+\frac{1}{2}}^{\text {ext }}$
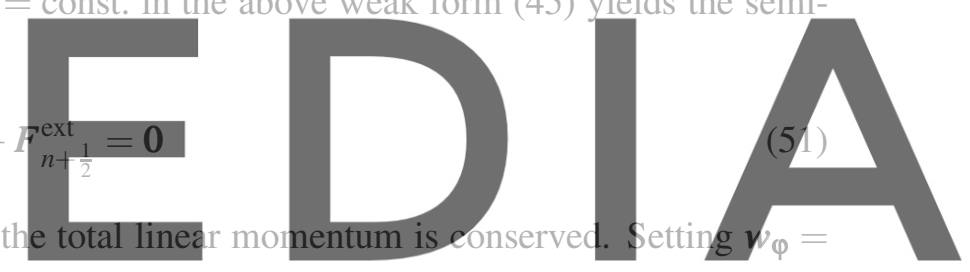

$\xi \times \varphi_{n+\frac{1}{2}}$ with $\xi=$ const. in (45) yields the balance of angular momentum

Register for free at https//www.scipedia.com to download the version without the watermark

$$
\frac{\Delta J}{\Delta t}-M_{n+\frac{1}{2}}^{\mathrm{ext}}=0
$$

Hence, for vanishing external torque $\boldsymbol{M}_{n+\frac{1}{2}}^{\text {ext }}$ the total angular momentum is preserved. Setting $w_{\varphi}=\xi=$ const. in (46) leads to the global form of Gauß law

$$
\int_{\mathcal{B}_{0}} \rho_{0_{n+1 / 2}}^{e} \mathrm{~d} V+\int_{\partial_{\omega} \mathcal{B}_{0}} \omega_{0_{n+1 / 2}}^{e} \mathrm{~d} A=0
$$

Considering of time independent forces and electric charges, setting the test functions $\left\{\boldsymbol{w}_{\boldsymbol{v}}, \boldsymbol{w}_{\boldsymbol{\varphi}}, w_{\phi}, \boldsymbol{w}_{\boldsymbol{D}}\right.$, $\left.w_{\theta}\right\}=\left\{\frac{\Delta v}{\Delta t}, \frac{\Delta \phi}{\Delta t}, \frac{\Delta \varphi}{\Delta t}, \frac{\Delta D}{\Delta t}, 1\right\}$ in (44)-(48) and summing them up yields, after some algebra, the desired balance of energy

$$
\frac{\Delta K}{\Delta t}+\int_{\mathcal{B}_{0}} \frac{\Delta(W+\boldsymbol{D} \cdot \nabla \varphi+\theta \eta)}{\Delta t} d V-\frac{\Delta \Pi_{\mathrm{ext}}^{\mathrm{m}}(\boldsymbol{\varphi})}{\Delta t}-\frac{\Delta \Pi_{\mathrm{ext}}^{\mathrm{e}}(\phi)}{\Delta t}-Q_{\mathrm{ext}}=0
$$

Accordingly, the total energy is conserved if the external and thermal power vanishes. 


\section{Finite element approximation}

The semi-discrete system given in (44)-(48) is discretized in space employing the isoparametric finite element method. In this connection, the geometry is subdivided into a finite number of elements $n_{\mathrm{el}}$, such that $\mathcal{B}_{0} \approx \mathcal{B}_{0}^{\mathrm{h}}=\cup_{e}^{n_{\mathrm{el}}} \mathcal{B}_{0_{e}}^{\mathrm{h}}$. The unknown fields in the semi-discrete weak forms in (44)-(48) are discretized employing appropriate functional spaces such that $(\bullet)^{\mathrm{h}} \in \mathbb{V}^{(\bullet)^{\mathrm{h}}} \subset \mathbb{V}^{(\bullet)}$ where $(\bullet)$ represents the unknowns $\{\boldsymbol{v}, \boldsymbol{\varphi}, \boldsymbol{\phi}, \boldsymbol{D}, \theta\}$, respectively. In particular, solution and test functions are discretized following a BubnovGalerkin approach, i.e.

$$
(\bullet)^{\mathrm{h}}(\boldsymbol{X})=\sum_{a=1}^{n(\bullet)} N_{a}^{(\bullet)}(\boldsymbol{X})(\bullet)_{a}(t), \quad w_{(\bullet)}^{\mathrm{h}}(\boldsymbol{X})=\sum_{a=1}^{n(\bullet)} N_{a}^{(\bullet)}(\boldsymbol{X}) w_{(\bullet)_{a}}(t)
$$

where the $a$-th shape function $N_{a}(\boldsymbol{X})$ are associated with the nodal quantities $(\bullet)_{a}(t)$ with $a=1,2, \ldots, n_{(\bullet)}$ for the approximation of the respective field $(\bullet)$. Furthermore, $n_{(\bullet)}$ denotes the number of nodes per element of the discretisation associated with the field $(\bullet)$. Different interpolation orders can be applied, which should be in accordance with the stability (inf-sup) condition (cf. [15]). Using discontinuous shape functions for the solution and test spaces of $\boldsymbol{D}$, a static condensation reduces the size of the equation system, which yields computational costs comparable to a minimal four field approach $\{\boldsymbol{v}, \boldsymbol{\varphi}, \phi, \theta\}$. It can be shown that the applied spatial discretization does not affect the balance laws given in Sect. 5 . Accordingly, the proposed EM scheme inherits the balance laws for the full discrete system.

8 Computational examples

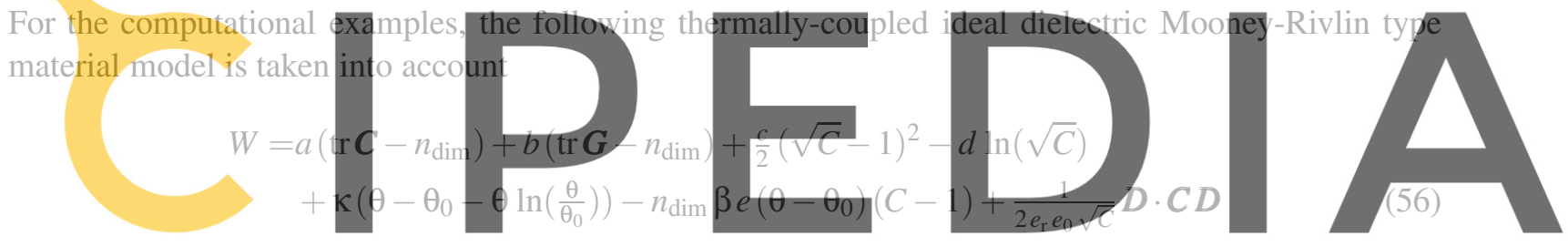

where the material parameters are introduced in Tab. 1. Spatial discretisation is employed with $H 2^{c} H 1^{d}$ -

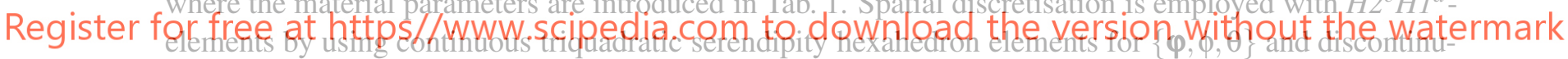
ous trilinear hexahedron elements for $\{\boldsymbol{D}\}$.

$\begin{array}{lccl}\text { mechanical parameters } & a, b, c, e & & \\ \text { coupling coefficient } & \beta & 2.233 \cdot 10^{-4} & \mathrm{~K}^{-1} \\ \text { reference temperature } & \theta_{0} & 293.15 & \mathrm{~K} \\ \text { electrical parameters } & \varepsilon_{0} & 8.854 \times 10^{-12} & \mathrm{~A}^{2} \mathrm{~s}^{4} \mathrm{~kg}^{-1} \mathrm{~m}^{-3} \\ & \varepsilon_{r} & 4 & - \\ \text { reference potential } & \varphi_{0} & 0 & \mathrm{~V}\end{array}$

Table 1: Material parameters.

\subsection{Static example}

The convergence analysis of an ad hoc manufactured problem is carried out next (cf. [7, 14]) by neglecting time effects. 


$\begin{array}{lccl}\text { mechanical parameters } & a, b, c, e & 1 & \mathrm{~Pa} \\ \text { specific heat capacity } & \kappa & 1 & \mathrm{JK}^{-1} \mathrm{~m}^{-3} \\ \text { thermal conductivity } & k_{0} & 0.1 & \mathrm{WK}^{-1} \mathrm{~m}^{-1} \\ \text { reference Temperature } & \theta_{0} & 293.15 & \mathrm{~K} \\ \text { Mass density } & \rho_{0} & 0 & \mathrm{kgm}^{-3} \\ \text { Newton tolerance } & \varepsilon & 1 \cdot 10^{-6} & - \\ \text { simulation duration } & T & 1 & \mathrm{~s} \\ \text { increment size } & \Delta t & 0.05 & \mathrm{~s}\end{array}$

Table 2: Material and simulation parameters (note that $d=2(a+2 b)$ ) for the static example.

A body $\mathcal{B}_{0}(0,1)[\mathrm{m}] \times(0,1)[\mathrm{m}] \times(0,1)[\mathrm{m}]$ is considered, where material and simulation parameters are provided in Tab. 2. Furthermore, the exact fields

$$
\varphi^{\text {exact }}=X+\gamma_{1} \sin (X) e_{1}+\gamma_{2} \cos (Y) e_{2}+\gamma_{3}(\sin (Z)+\cos (Z)) e_{3}, \quad \phi^{\text {exact }}=\tilde{\varphi}_{0} X, \quad \theta^{\text {exact }}=\tilde{\theta}_{0} Y
$$

are assumed, where $\gamma_{i}=\frac{i}{2} e-1, i=1,2,3, \tilde{\varphi}_{0}=1 e 2$ and $\tilde{\theta}_{0}=1 e 1$ (cf. [7]). With the above, exact values for $\boldsymbol{F}, \boldsymbol{C}, \boldsymbol{G}, \boldsymbol{C}, \boldsymbol{E}, \boldsymbol{D}, \boldsymbol{Q}$ and $\boldsymbol{S}$ are computed. Moreover, with the balance equations exact values for $\boldsymbol{B}$, $\rho_{0}$ and $R$ are computed and imposed within the inner body for a numerical study. The exact solutions (57) are imposed as a Dirichlet boundary at the surfaces of the body. Eventually, exact solutions (57) are compared against the numerical solutions. In particular, the h-convergence rate for the unknowns

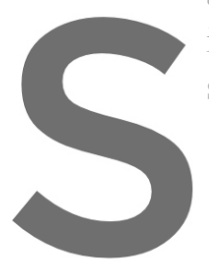
is computed by employing the $L^{2}$-norm of the entor
solutions and the convergence results for all unknow
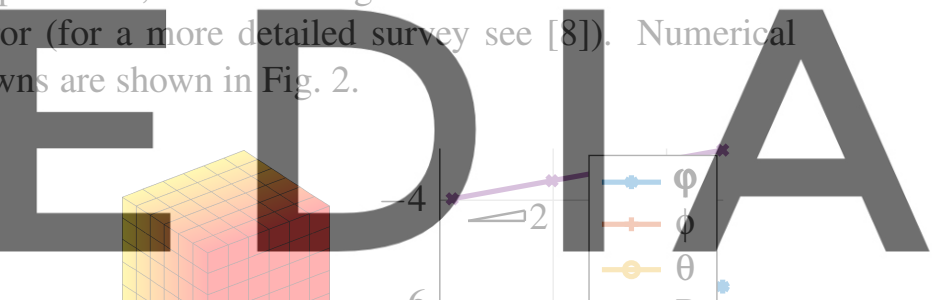

Register for free at https//www.scipedia.com to do (b)

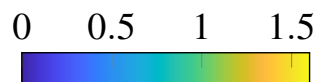

$\begin{array}{llllll}0 & 20 & 40 & 60 & 80 & 100\end{array}$

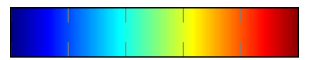

download the version without the watermark

(c)

295

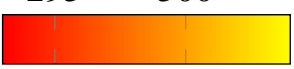

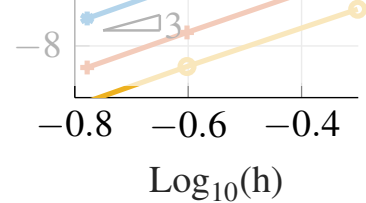

Figure 2: Von Mises stress (a), electrical potential (b) and thermal field (c). Convergence analysis (right).

Obviously, a $p+1$ convergence in all variables for the $H 2^{c} H 1^{d}$ element (see Fig. 2) can be observed.

\subsection{Transient example}

An L-shaped body, depicted in Fig. 3 is considered (cf. [6]) to be free in space. Material and simulation parameters as well as the geometry of the body are presented in Tab. 3. As indicated in Fig. 3 (right), two faces of the block are subjected to initial temperature conditions. Time-dependent electrical Dirichlet boundaries and pressure loads are applied as follows (see Fig. 3, left) For the applied $H 2^{c} H 1^{d}$ elements, 
a linear temperature distribution is assumed within the first face elements in direction of the bulk, as can be observed for the first snapshot in Fig. 3 .

$$
\begin{gathered}
\varphi_{1}(X, Y=-1.5, Z)=0, \quad \varphi_{2}(X, Y=0, Z)= \begin{cases}6 e 6 \sin \left(\frac{\pi}{2} \frac{t}{5}\right) & \text { for } t \leq 5 \mathrm{~s} \\
6 e 6 & \text { for } t>5 \mathrm{~s}\end{cases} \\
\boldsymbol{P}_{1}(t)=-\boldsymbol{P}_{2}(t)=f(t)\left(\begin{array}{l}
256 / 9 \\
512 / 9 \\
768 / 9
\end{array}\right) \frac{\mathrm{N}}{\mathrm{m}^{2}}, \quad \text { with } \quad f(t)= \begin{cases}t & \text { for } t \leq 2.5 \mathrm{~s} \\
5-t & \text { for } 2.5 \leq t \leq 5 \mathrm{~s} \\
0 & \text { for } t>5 \mathrm{~s}\end{cases}
\end{gathered}
$$

Exemplary, snapshots of the motion with corresponding thermal field are depicted in Fig. 3. After the loading phase, only a constant electrical potential field is applied such that for this isolated system with $t>5 \mathrm{~s}$ the total linear momentum, angular momentum and energy are conserving quantities, respectively. In Figs. 4 and 5 the total angular momentum and energy evolution of both, the EM consistent and a midpoint (MP) integrator are shown (for a more detailed survey see [8]).
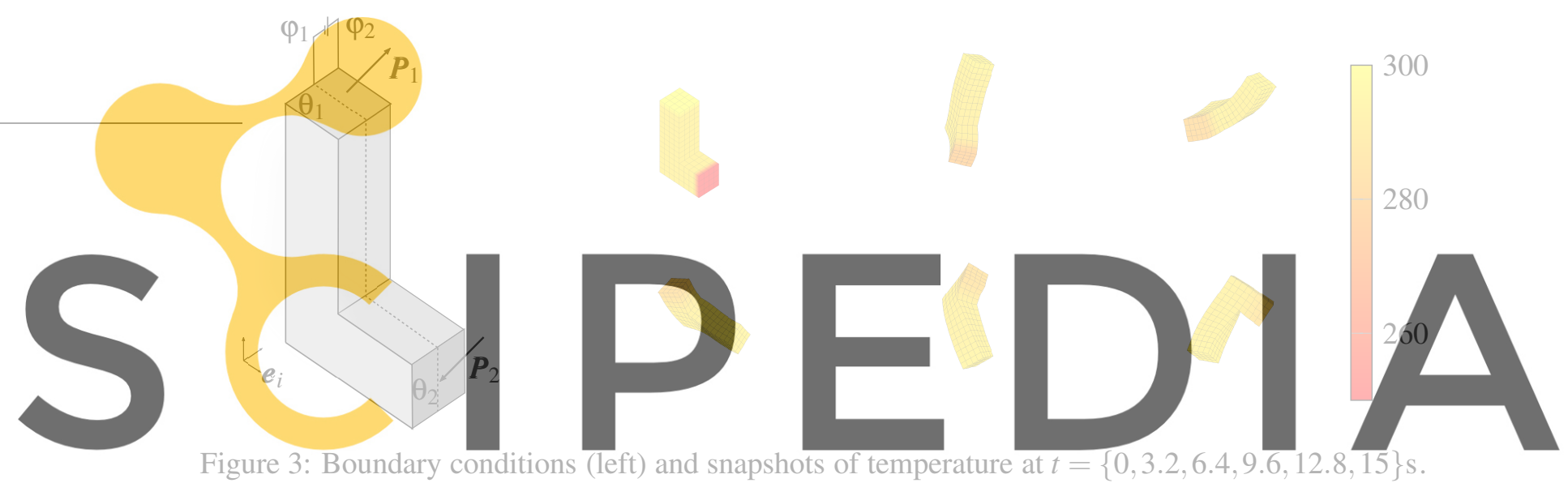

\section{Register for free at https//www.scipedia.com to download the version without the watermark}

The EM scheme correctly reproduces both the total angular momentum and the energy, whereas the MP integrator becomes unstable accompanied by an energy blow-up. Furthermore, the order of accuracy of the EM integration scheme is elaborated via the $L_{2}$-norm of the error computed for the time interval after the loading phase, i.e. $5 \leq t \leq 5.1$. Fig. 6 shows second order accuracy in time of the EM integration scheme. Accordingly, the conserving properties, the second order accuracy and the superior robustness properties are shown when compared to a standard integration scheme.

\section{CONCLUSIONS}

A new one-step implicit and consistent EM integration scheme has been proposed for the simulation of thermo-electro-elastodynamics employing finite deformations, thermal and electrical fields. The design of the EM scheme is facilitated by both the tensor cross product and polyconvexity inspired constitutive models. The introduced examples show second order accuracy in time and structure preserving, i.e. energy and momentum consistent, behavior. Eventually, the superior numerical robustness and stability properties of the EM scheme have been demonstrated when compared to a standard midpoint rule (for a more detailed overview it is referred to [8]). 
Table 3: Material and simulation parameters and geometry.

$\begin{array}{lccl}\text { mechanical parameters } & a & 831.2500 & \mathrm{~Pa} \\ & b & 166.2500 & \mathrm{~Pa} \\ & c & 0 & \mathrm{~Pa} \\ & e & 1000 & \mathrm{~Pa} \\ \text { specific heat capacity } & \kappa & 100 & \mathrm{JK}^{-1} \mathrm{~m}^{-3} \\ \text { thermal conductivity } & k_{0} & 10 & \mathrm{WK}^{-1} \mathrm{~m}^{-1} \\ \text { density } & \rho_{0} & 100 & \mathrm{kgm}^{-3} \\ \text { initial temperatures } & \theta_{1}, \theta_{2} & 300,250 & \mathrm{~K} \\ \text { Newton tolerance } & \varepsilon & 10^{-6} & - \\ \text { simulation duration } & T & 100 & \mathrm{~S} \\ \text { time step size } & \Delta t & 0.8 & \mathrm{~S}\end{array}$

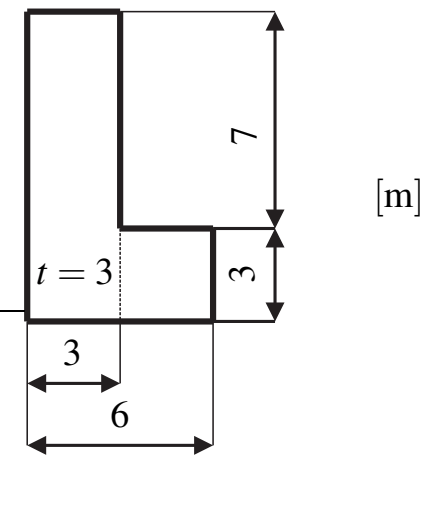

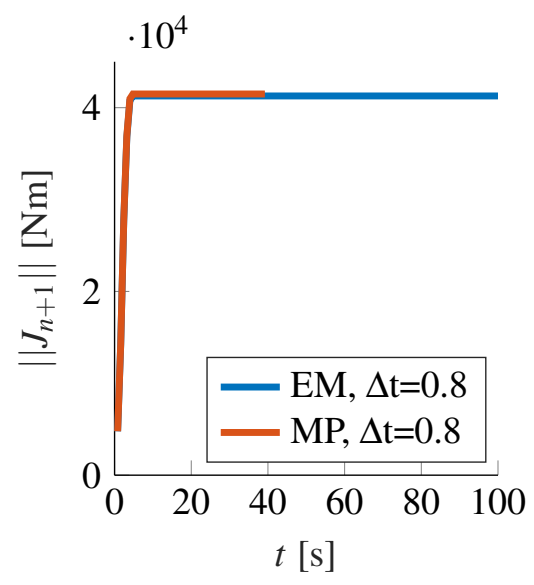

Figure 4: Angular momentum.

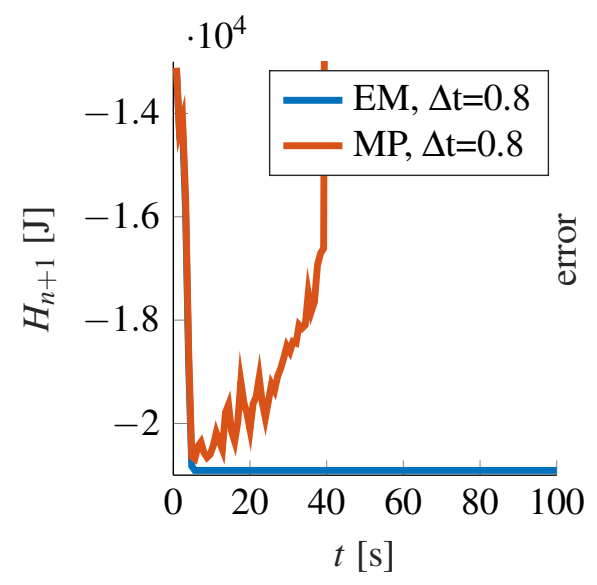

Figure 5: Energy.

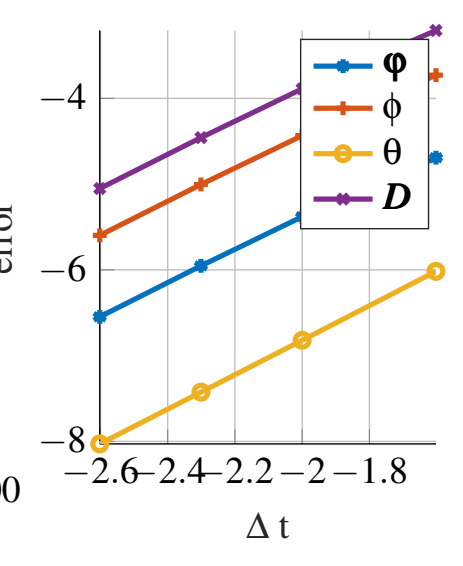

Figure 6: Study of error.

\section{REFERENCES}

[1] P. Betsch, editor. Structure-preserving Integrators in Nonlinear Structural Dynamics and Flexible Multibody Dynamics, volume 565 of CISM Courses and Lectures. Springer-Verlag, 2016.

[2] P. Betsch, A. Janz, and C. Hesch. A mixed variational framework for the design of energy-momentum schemes inspired by the structure of polyconvex stored energy functions. Computer Methods in Applied Mechanics and Engineering, 335:660 - 696, 2018.

[3] J. Bonet, A. Gil, and R. Ortigosa. A computational framework for polyconvex large strain elasticity. Computer Methods in Applied Mechanics and Engineering, 283:1061 - 1094, 2015.

[4] R. de Boer. Vektor- und Tensorrechnung für Ingenieure. Springer-Verlag, 1982.

[5] A. Dorfmann and R. W. Ogden. Nonlinear electroelasticity. Acta Mechanica, 174(3-4):167-183, 2005. 
[6] M. Franke, A. Janz, M. Schiebl, and P. Betsch. An energy momentum consistent integration scheme using a polyconvexity-based framework for nonlinear thermo-elastodynamics. Int. J. Numer. Methods Eng., 115(5):549-577, 2018.

[7] M. Franke, R. Ortigosa, A. Janz, A. Gil, and P. Betsch. A mixed variational framework for the design of energy-momentum integration schemes based on convex multi-variable electroelastodynamics. Computer Methods in Applied Mechanics and Engineering, 351:109 - 152, 2019.

[8] M. Franke, R. Ortigosa, J. Martinez-Frutos, A. Gil, and P. Betsch. A thermodynamically consistent time integration scheme for non-linear thermo-electro-mechanics. In preparation, 2021.

[9] O. Gonzalez. Exact energy and momentum conserving algorithms for general models in nonlinear elasticity. Comput. Methods Appl. Mech. Engrg., 190:1763-1783, 2000.

[10] M. Mehnert, M. Hossain, and P. Steinmann. Numerical modeling of thermo-electro-viscoelasticity with field-dependent material parameters. International Journal of Non-Linear Mechanics, 106:1324, 2018.

[11] R. Ortigosa, M. Franke, A. Janz, A. Gil, and P. Betsch. An energy-momentum time integration scheme based on a convex multi-variable framework for non-linear electro-elastodynamics. Comput. Methods Appl. Mech. Engrg., 339:1 - 35, 2018.

[12] R. Ortigosa, A. Gil, J. Martínez-Frutos, M. Franke, and J. Bonet. A new energy-momentum time integration scheme for non-linear thermo-mechanics. Computer Methods in Applied Mechanics and Engineering, 372:113395, 2020.

[13] R. Pelrine, R. Kornbluh, Q. Pei, and J. Joseph. High-speed electrically actuated elastomers with strain greater than $100 \%$. Science, 287(5454):836-839, 2000.

[14] R. Poya, A. J. Gil, R. Ortigosa, R. Sevilla, J. Bonet, and W. A. Wall. A curvilinear high order finite element framework for electromechanics: From linearised electro-elasticity to massively deformable dielectric elastomers. Comput. Methods Appl. Mech. Engrg., 329:75-117, 2018.

[15] J. Schröder, P. Wriggers, and D. Balzani. A new mixed finite element based on different approximations of the minors of deformation tensors. Comput. Methods Appl. Mech. Engrg., 200(4952):3583-3600, 2011.

[16] Z. Suo, X. Zhao, and W. H. Greene. A nonlinear field theory of deformable dielectrics. Journal of the Mechanics and Physics of Solids, 56(2):467-486, 2008.

[17] R. Vertechy, G. Berselli, C. V. P., and M. Bergamasco. Continuum thermo-electro-mechanical model for electrostrictive elastomers. Journal of Intelligent Material Systems and Structures, 24(6):761-778, 2013.

[18] F. Vogel, R. Bustamante, and P. Steinmann. On some mixed variational principles in electroelastostatics. International Journal of Non-Linear Mechanics, 47(2):341-354, 2012. Nonlinear Continuum Theories.

[19] D. K. Vu and P. Steinmann. On 3-D coupled BEM-FEM simulation of nonlinear electroelastostatics. Comput. Methods Appl. Mech. Engrg., 201-204(0):82-90, 2012. 